\title{
Formation of Covalently Bonded Polycyclic Aromatic Hydrocarbons in the Interstellar Medium
}

\author{
Tao Chen ${ }^{1,2}$ (iD) \\ ${ }^{1}$ Leiden University, Leiden Observatory, Niels Bohrweg 2, NL-2333 CA Leiden, Netherlands; chen@strw.leidenuniv.nl \\ ${ }^{2}$ School of Engineering Sciences in Chemistry, Biotechnology and Health, Department of Theoretical Chemistry \& Biology, \\ Royal Institute of Technology, SE-10691, Stockholm, Sweden \\ Received 2018 August 23; revised 2018 September 20; accepted 2018 September 20; published 2018 October 19
}

\begin{abstract}
Photo-/ion-induced ionization and dissociation processes are commonly observed for polycyclic aromatic hydrocarbon (PAH) molecules. This work performs theoretical studies of PAHs and their fragments. Molecular dynamics simulations in combination with static quantum chemical calculations reveal that following a single hydrogen atom loss, the fragments, $\mathrm{PAH}-\mathrm{H}$, are extremely reactive. They catch a neighbor molecule within picoseconds to form a covalently bonded large molecule regardless of orientations/angles and temperatures. We calculate the infrared spectra of the covalently bonded molecules, which indicate that such species could be the carrier of unidentified infrared emission bands. It also implies that regular PAHs might be less abundant in space than what is expected.
\end{abstract}

Key words: astrochemistry - ISM: molecules - infrared: ISM - line: formation - methods: laboratory: molecular

\section{Introduction}

The broad IR emission bands at $3.3 \mu \mathrm{m}, 6.2 \mu \mathrm{m}, 7.7 \mu \mathrm{m}$, $8.6 \mu \mathrm{m} 11.2 \mu \mathrm{m}$, and $12.7 \mu \mathrm{m}$ bands were discovered in 1973 (Leger \& Puget 1984; Allamandola et al. 1989). They are commonly observed in a variety of astronomical objects, ranging from the Galaxy to extragalactic regions (Tielens 2008). Due to the absence of spectroscopic identification and lack of excitation mechanisms, for a long time, these bands were called unidentified infrared emission features (UIE; Leger \& Puget 1984). Nowadays, these UIE bands are generally attributed to visible/UV photon-excited polycyclic aromatic hydrocarbon (PAH) molecules (Allamandola et al. 1989; Tielens 2008), in which the $3.3 \mu \mathrm{m}$ band is attributed to the $\mathrm{C}-\mathrm{H}$ stretching modes, the 6.2 and $7.7 \mu \mathrm{m}$ bands to the $\mathrm{C}-\mathrm{C}$ stretching modes, while 11.2 and $12.7 \mu \mathrm{m}$ are attributed to the $\mathrm{C}-\mathrm{H}$ out-of-plane bending modes of PAHs (Tielens 2008). With their compact and stable structure, PAHs are believed to be abundant and ubiquitous in the interstellar medium (ISM), locking up $10 \%-20 \%$ of the elemental carbon (Tielens 2008).

In the ISM, a number of different reactions related to PAHs can take place: following the absorption of UV photons from luminous stars, most of the time, PAHs will relax through IR vibrational relaxation (Allamandola et al. 1989; Tielens 2008), but there is the probability of photofragmentation and loss of $\mathrm{H}\left(\mathrm{C}_{2} \mathrm{H}_{2}\right.$ fragments have been indicated as dominant loss channels for small PAHs; Ekern et al. 1998; Jochims et al. 1999; West et al. 2012; Zhen et al. 2014), isomerization (Dyakov et al. 2006; Johansson et al. 2011; Solano \& Mayer 2015; Simon et al. 2017; Trinquier et al. 2017), and ionization (Holm et al. 2011; Zhen et al. 2015). Large PAHs, on the other hand, may preferentially lose all of their $\mathrm{H}$, and the resulting graphene sheet may isomerize to fullerenes such as $\mathrm{C}_{60}$, which is very stable under interstellar conditions (Ekern et al. 1998; Berné \& Tielens 2012; Zhen et al. 2014).

Fragmentation turns out to be crucial for molecular growth processes in $\mathrm{PAH} /$ fullerene clusters; e.g., recent works have shown that following the loss of $\mathrm{C}$ atoms, $\mathrm{C}_{119}^{+}$and $\mathrm{C}_{118}^{+}$could be formed in collisions between $\mathrm{keV}$ alpha particles and van der Waals clusters of $\mathrm{C}_{60}$ fullerenes (Zettergren et al. 2013). In such experiments, a carbon atom or $\mathrm{C}_{2}$ is knocked out directly from a $\mathrm{C}_{60}$. The newly formed $\mathrm{C}_{59}^{+}$or $\mathrm{C}_{58}^{+}$radicals react with a neighbor $\mathrm{C}_{60}$ to form $\mathrm{C}_{119}^{+}$and $\mathrm{C}_{118}^{+}$within picoseconds (Seitz et al. 2013; Wang et al. 2014). A similar process has been observed in pyrene clusters following $\mathrm{C}$ and/or $\mathrm{H}$ losses (Delaunay et al. 2015) and in small hydrocarbon chain clusters (Gatchell et al. 2017) in which new molecules are formed following a direct knockout of atoms via Rutherford-like ionatom scattering processes.

The formation of large PAHs following photodissociation processes in PAH clusters has been reported recently (Zhen et al. 2018b). However, those studies are multiple-step reactions, e.g., several $\mathrm{H}$ losses and barriers are required in the the reactions (Zhen et al. 2018b), which can only be achieved through multiphoton processes (Montillaud et al. 2013; Andrews et al. 2016). Such formation processes might be less feasible in the ISM in comparison to single-photon processes, which are generally believed to be the carrier of UIE bands (Allamandola et al. 1985, 1989; Tielens 2008). In this work, we focus on exothermic and barrier-free reactions, which are accessible even in low-density and low-temperature astronomical environments. Due to the compact and stable structure, we choose the pyrene molecule for this study. Figure 1 shows three possible dissociation channels for $\mathrm{H}$ and $\mathrm{H}_{2}$ losses from a pyrene $\left(\mathrm{C}_{16} \mathrm{H}_{10}\right)$ molecule (Chen et al. 2015). The energy differences among these channels are rather small, e.g., for $\mathrm{H}$ losses, the lowest adiabatic dissociation energy is $5.09 \mathrm{eV}$ ( $\mathrm{H} a$ in Figure 1), while the highest one is $5.33 \mathrm{eV}(\mathrm{Hc}$ in Figure 1; Chen et al. 2015). $\mathrm{H}_{2}$ losses are competing processes against $\mathrm{H}$ loss from a PAH molecule; however, it involves several barriers, e.g., one or two barriers for hydrogen migrations and one barrier for $\mathrm{H}_{2}$ dissociate from a PAH (Chen et al. 2015), in which the highest barriers are comparable with the dissociation energy of $\mathrm{H}$ losses (Chen et al. 2015). This article investigates several possible formation pathways, structures, and IR features of pyrene and pyrene- $\mathrm{H} /$ pyrene-2H. The dynamical formation processes and instant structures are studied using ab initio molecular dynamics simulations. The 

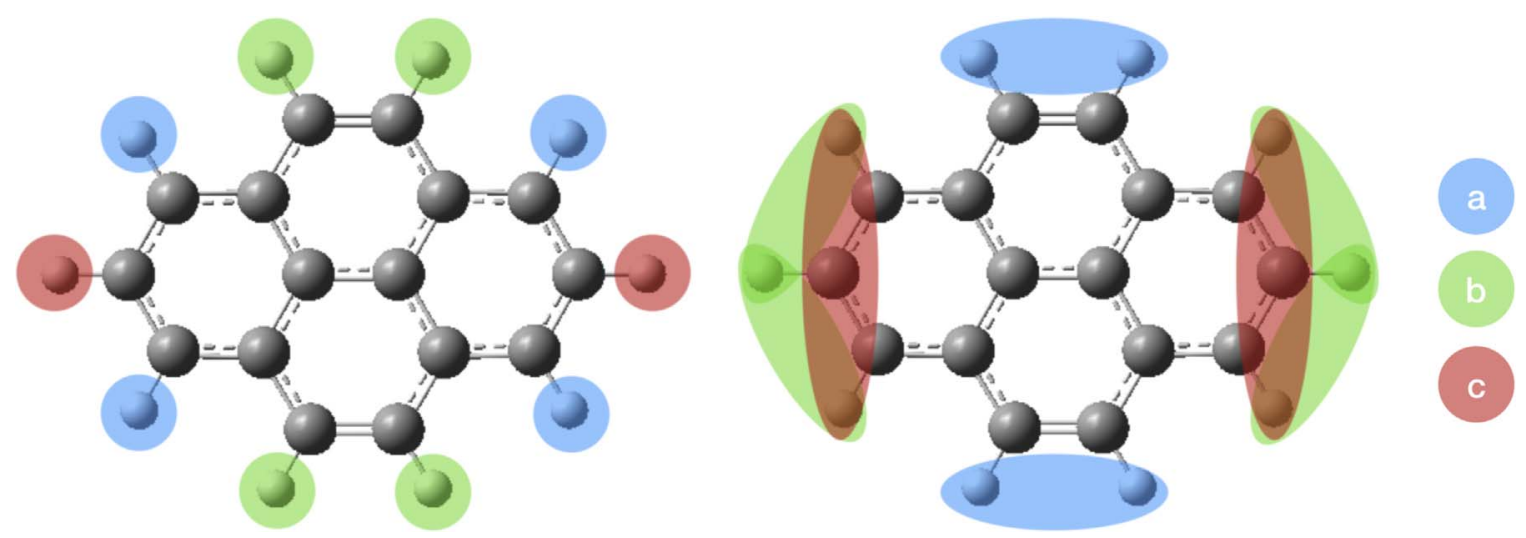

Figure 1. Three possible dissociation channels for the losses of $\mathrm{H}$ and $\mathrm{H}_{2}$ from pyrene $\left(\mathrm{C}_{16} \mathrm{H}_{10}\right)$. For $\mathrm{H}$ losses, hydrogens with the same color are identical in terms of dissociation energy. For $\mathrm{H}_{2}$ losses, two hydrogens are linked using the same color; again, the $\mathrm{H}_{2}$ with the same color are identical in energy.

Table 1

The Binding Energies and Geometries for the Benzene $\left(\mathrm{C}_{6} \mathrm{H}_{6}\right)$ Dimer

\begin{tabular}{|c|c|c|c|c|}
\hline Method & Binding energy $(\mathrm{kcal} / \mathrm{mol})^{\mathrm{a}}$ & Dimer distance $(\AA)^{\mathrm{b}}$ & $\mathrm{C}-\mathrm{C}$ bond $(\AA)^{\mathrm{c}}$ & $\mathrm{C}-\mathrm{H}$ bond $(\AA)$ \\
\hline B3LYP-D3/3-21G* & 3.97 & 3.90 & 1.398 & 1.084 \\
\hline B3LYP-D3/6-311++G(2d,p) & 2.96 & 3.95 & 1.392 & 1.084 \\
\hline Sinnokrot et al. (2002) & 2.78 & 3.76 & $\ldots$ & $\ldots$ \\
\hline Park \& Lee (2006) & 3.03 & 3.94 & 1.395 & 1.087 \\
\hline
\end{tabular}

Notes.

${ }^{\mathrm{a}}$ The energies are measured for two parallel-displaced benzenes.

${ }^{b}$ The distances are measured between the geometrical center of two parallel-displaced benzenes.

${ }^{c}$ The bond lengths are measured for benzene monomers.

ground-state structures and IR features are calculated with static quantum chemical calculations.

\section{Computational Details}

Theoretical calculations are carried out using density functional theory (DFT) with the hybrid density functional B3LYP (Lee et al. 1988; Becke 1992) as implemented in the Gaussian 16 program (Frisch et al. 2016). All structures are optimized using the $6-311++\mathrm{G}(2 \mathrm{~d}, \mathrm{p})$ basis set. The vibrational frequencies are calculated for the optimized geometries to verify that these correspond to minima or first-order saddle points (transition states) on the potential energy surface (PES). We have taken the zero-point vibrational energy (ZPVE) into account. The ZPVE values are scaled by the empirical factor 0.965 to correct for anharmonic effects (Andersson \& Uvdal 2005). In all cases, we have only considered the ground-state PES as the non-radiative decay for such a large molecule is very rapid: $\sim 10^{-12} \mathrm{~s}$ (Vierheilig et al. 1999; Zewail 2000). Only the lowest spin state is considered, which seems to be a well-justified approach to predict reasonable energies in comparison to the experimental results (Holm et al. 2011). The dispersion-corrected B3LYP-D3 (Grimme et al. 2011) is considered to account for the intermolecular forces in PAH clusters.

The dynamical processes are simulated using the Atom Centered Density Matrix Propagation (ADMP) molecular dynamics model (Iyengar et al. 2001; Schlegel et al. 2001, 2002). This method provides equivalent functionality to BornOppenheimer molecular dynamics at a considerably reduced computational cost (Schlegel et al. 2002). The functional of B3LYP in combination with the basis sets of $3-21 \mathrm{G}^{*}$ is utilized for the simulations. To take the weakly bonded systems into account, we consider the dispersion-corrected B3LYP-D3. To evaluate the accuracies of the functional and basis sets, the benzene dimer has been investigated. Table 1 shows the binding energies and geometries of the parallel-displaced benzene dimer. Moreover, the bond distances of the benzene monomer and the corresponding values from the literature (Sinnokrot et al. 2002; Tsuzuki et al. 2002; Park \& Lee 2006) are also presented. One can see that B3LYP-D3/3-21G* produces a slightly higher value of the binding energy, but provides rather accurate geometries in comparison to other high-level calculations. This work focuses on the geometry changes in the dynamical system of PAH clusters; B3LYP-D3 / $3-21 \mathrm{G}^{*}$ ought to be sufficient for such a study.

For the ADMP calculations, a step size of $0.3 \mathrm{fs}$ was used for each system. The initial structure/positions of the molecules were optimized before running the molecular dynamics simulations. The initial velocity of each molecule was set to zero.

\section{Results and Discussion}

It has been shown that $\mathrm{H}$ and $\mathrm{H}_{2}$ losses from PAHs are efficient fragmentation pathways for both ion-induced and photoinduced experiments (Dyakov et al. 2006; West et al. 2014; Chen et al. 2015).

To study the reactivity of pyrene-Hx $(x=1$ or 2$)$, we performed molecular dynamics simulations. The charged systems, i.e., pyrene + pyrene-Hx, which are positively charged, are investigated first. Figure 2 shows five snapshots from the molecular dynamics simulations. The initial geometry comprises an intact pyrene in combination with a pyrene-Ha 

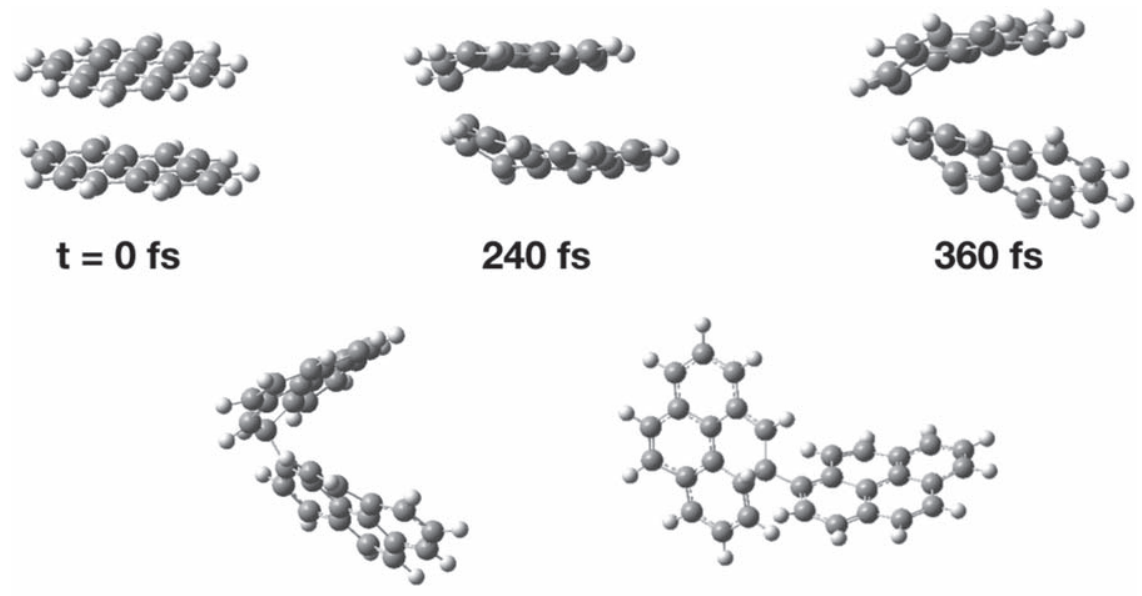

$480 \mathrm{fs}$

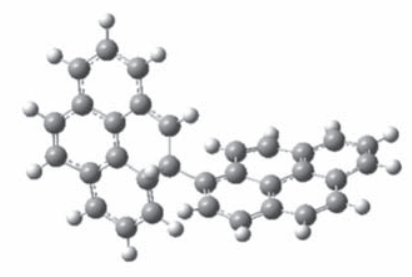

\section{$720 \mathrm{fs}$}

Figure 2. Five snapshots from molecular dynamics simulations showing the interaction between pyrene and pyrene-Ha (see Figure 1). A covalently bonded pyrene complex is formed without any external energy or initial velocity within a picosecond.

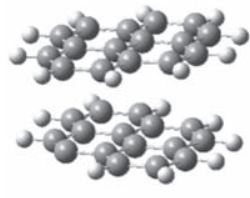

$\mathbf{t}=\mathbf{0}$ fs

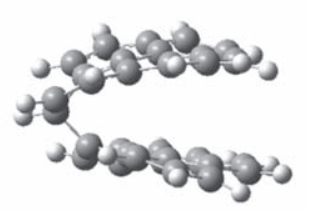

$240 \mathrm{fs}$

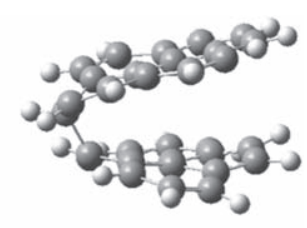

$360 \mathrm{fs}$

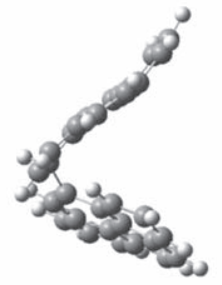

1200 fs

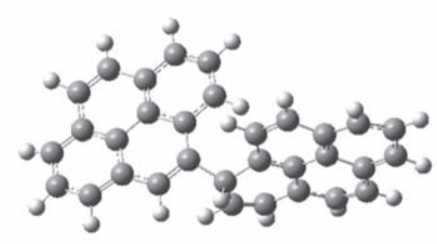

1800 fs

Figure 3. Five snapshots from molecular dynamics simulations showing the interaction between pyrene and pyrene-Hb (see Figure 1). A covalently bonded pyrene complex is formed without any external energy or initial velocity within a picosecond.

molecule (see Figure 1), which are oriented in a parallel position (stacked) and separated by $\sim 3.5 \AA$ (this value is the ground-state distance for van der Waals complexes found via time-independent DFT calculations at $0 \mathrm{~K}$ ). It has been reported that for PAH clusters, the stacked structure corresponds to the most stable structure (Rapacioli et al. 2005).

The simulation shows that the molecules move close to each other rapidly in the beginning of the simulation, especially on the side where the hydrogen is lost, i.e., pyrene-Ha transform to a bent structure from a planar structure (see the second frame at $240 \mathrm{fs}$ in Figure 2). Subsequently (the third frame at $360 \mathrm{fs}$ in Figure 2), pyrene-Ha transforms back to a planar structure. However, an angled system is formed with an angle of $\sim 60^{\circ}$. The distance between the carbon atom (in pyrene-Ha) where $\mathrm{Ha}$ is located and the closest carbon atom in the intact pyrene remain almost unchanged $(\sim 1.65 \AA)$. At 480 fs into the simulation, the angle between the molecules increases, and a covalent bond is formed between the molecules, with a bond length of $\sim 1.55 \AA$. Finally, the molecules form a covalently bonded perpendicular system with a bond length of $\sim 1.5 \AA$, and they do not break or transform into other isomers until the end of the simulation (a few tens of picoseconds), i.e., the fifth frame in Figure 2 ought to be an equilibrated state/structure. We investigate different initial conditions, e.g., angle/orientation, distance, and temperature. It turns out that the formation of covalently bonded systems is very sensitive to the distance between molecules in comparison to the angle/orientation or temperature (see the discussion below for more details regarding the importance of the initial distance). If the initial geometry is set to a perpendicular orientation, or even at a low temperature (e.g., $4 \mathrm{~K}$ ), the covalently bonded system will always be formed in the simulation. See the time-independent DFT calculations below for the reason for the independence of the initial orientation and temperature.

Similar reaction pathways have been observed for the pyrene and the pyrene-Hb molecule system (see Figure 1). However, the formation of the covalently bonded perpendicular system, i.e, the equilibrated state, is about twice slower than for the case of pyrene-Ha, and the formed structure is slightly different; see Figure 3 for details. 


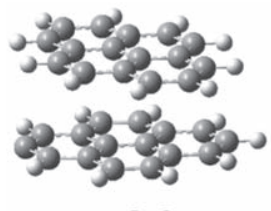

$t=0$ fs
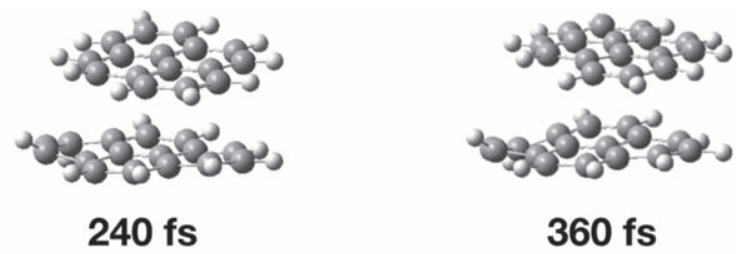

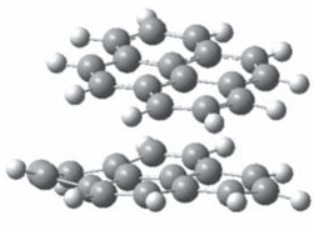

$1200 \mathrm{fs}$

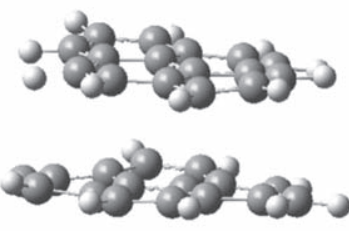

4800 fs

Figure 4. Five snapshots from molecular dynamics simulations showing the interaction between pyrene and pyrene-Hc (see Figure 1). No covalently bonded system is formed within 5 picoseconds.

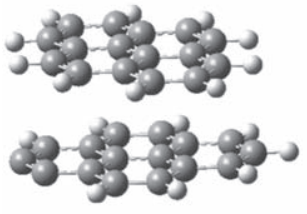

$\mathbf{t}=\mathbf{0}$ fs

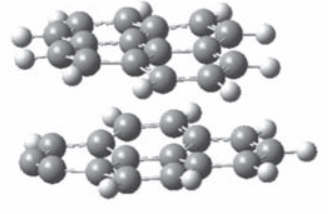

$600 \mathrm{fs}$

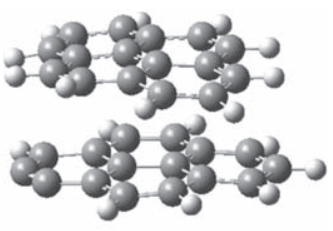

1200 fs

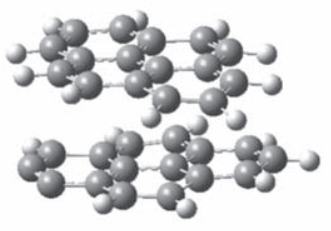

3000 fs

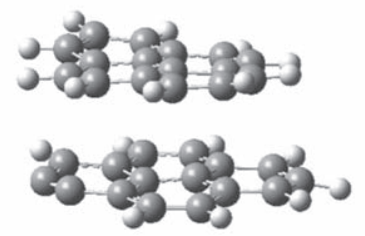

$3600 \mathrm{fs}$

Figure 5. Five snapshots from molecular dynamics simulations showing the interaction between pyrene and pyrene-2Ha (see Figure 1). No covalently bonded system is formed within 4 picoseconds.

For the case of pyrene-Hc, no covalently bonded system is formed within the simulation time. Instead, a weakly bonded van der Waals dimer is formed with a separation of $\sim 3.5 \AA$. See Figure 4 for details. We do not know why no covalently bonded molecule formed in such a case; it might be related to the fact that pyrene-Hc is a symmetric structure, which could reduce the reactivity of the radical.

We also study the neutral system, i.e., both molecules are neutral. However, for all possible single $\mathrm{H}$-loss positions, none of the covalently bonded systems was formed; instead, weakly bonded van der Waals dimers are formed with a structure similar to that in Figure 4. In other words, the charge plays a vital role in the formation of covalently bonded large molecules.

The loss of molecular hydrogens $\left(\mathrm{H}_{2}\right)$ is a dominant dissociation channel in statistical fragmentation processes (Dyakov et al. 2006; West et al. 2014; Chen et al. 2015). As shown in our previous study, three $\mathrm{H}_{2}$ loss pathways are the most energetically favorable (Chen et al. 2015); see Figure 1 for the positions. We study the reactivity of these three isomers for both neutral and charged systems. However, none of the covalently bonded systems was formed; only weakly bonded van der Waals dimers are formed. See Figure 5 for an example of the structure (pyrene + pyrene-2Ha).

To clarify the essence of the reactions, we carried out static quantum chemical calculations. Figure 6 shows the calculated sequential $\mathrm{H}$ losses from pyrene in combination with pyrene- $\mathrm{H}$. It turns out that the reaction between pyrene- $\mathrm{H}$ and pyrene is an exothermic reaction, i.e., there are attractive forces between these two species. It explains why the covalent bond is formed promptly as shown in the molecular dynamics simulations. In addition, we found that the dissociation energies of the hydrogens in between the molecules are rather low: $1.8-2.6 \mathrm{eV}$ in comparison to $\sim 5 \mathrm{eV}$ for a regular PAH. These results indicate that the molecules tend to form a complex larger molecule at first, then lose all of the aliphatic hydrogens in between to form a planar PAH (Zhen et al. 2018b). It is important to note that transition states are involved for the formations and not shown in Figure 6. Therefore, large planar PAHs are even more difficult to form in reality in comparison to covalently bonded systems, as shown in Figure 6 .

Figure 7 compares the calculated infrared spectra of a few molecules studied above and the observed IR spectrum of NGC 2023 and Orion Bar. The observational data are retrieved from the Infrared Space Observatory archive. The harmonic 


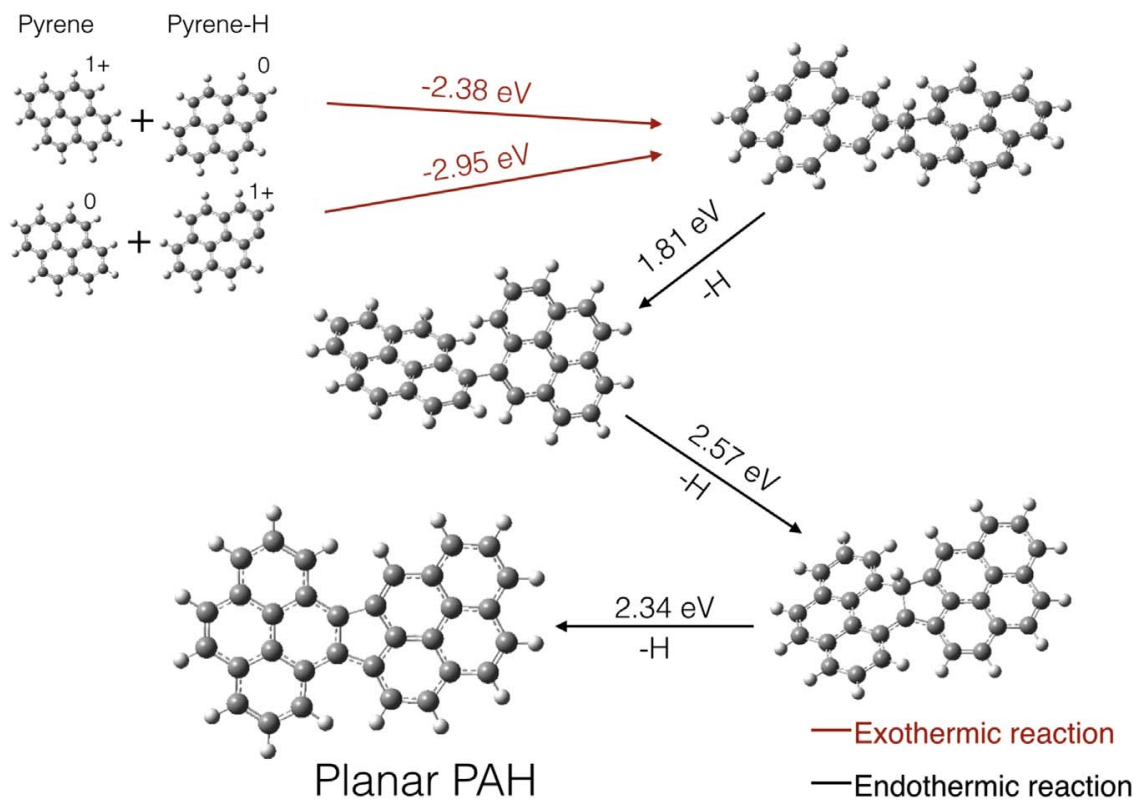

Figure 6. Calculated possible reaction pathways using B3LYP-D3/6-311++G(2d,p). The red arrows correspond to exothermic reactions, and the black arrows indicate endothermic reactions. The values on the arrows correspond to the dissociation energies of $\mathrm{H}$ losses or binding energies for the exothermic reactions.

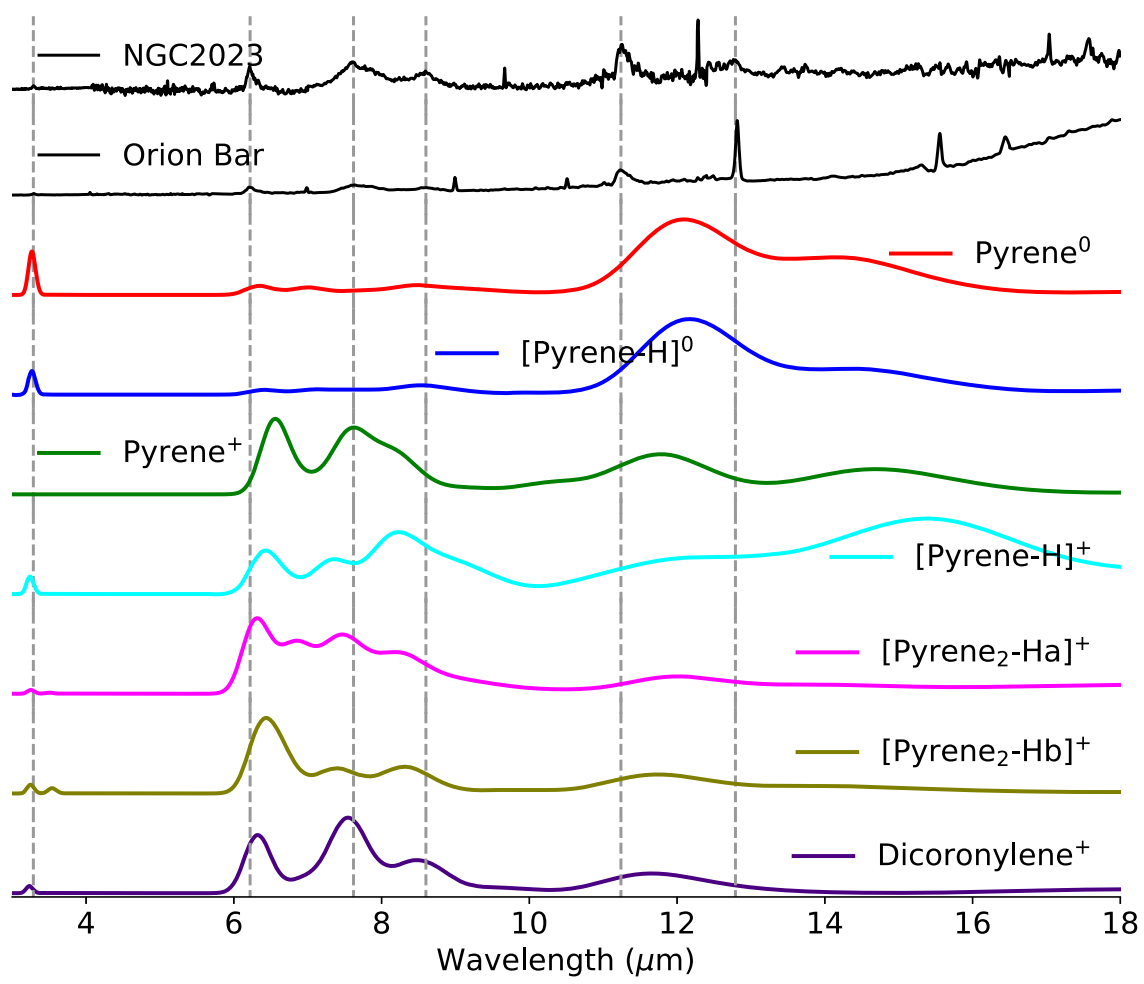

Figure 7. Observational IR spectrum of NGC 2023 and the Orion Bar, and calculated harmonic vibrational spectra of neutral and charged pyrene and pyrene-H, and covalently bonded pyrene, pyrene- $\mathrm{H}$, and dicoronylene cation. The calculated spectra are scaled by 0.965 and broadened by $45 \mathrm{~cm}^{-1}$ to correct for anharmonic and temperature effects. The dashed lines represent some dominant bands that are commonly observed in the ISM (Tielens 2008).

vibrational spectra are calculated using B3LYP/6-311++G (2d,p). The calculated spectra are further scaled by 0.965 and broadened by $45 \mathrm{~cm}^{-1}$ to correct for anharmonic and temperature effects. Several prominent bands, e.g., the $3.3 \mu \mathrm{m}, 6.2 \mu \mathrm{m}, 7.7 \mu \mathrm{m}, 8.6 \mu \mathrm{m} 11.2 \mu \mathrm{m}$, and $12.7 \mu \mathrm{m}$ bands, are marked with dashed lines. It is clear that the $3.3 \mu \mathrm{m}$ band can be well explained by the $\mathrm{C}-\mathrm{H}$ stretching of neutral PAHs, e.g., pyrene ${ }^{0}$ and $[p y r e n e-H]^{0}$, as well as the covalently bonded system, i.e., [pyrene $\left.{ }_{2}-\mathrm{Ha}\right]^{+}$and $\left[\text {pyrene }_{2}-\mathrm{Hb}\right]^{+}$. For the pyrene cation, i.e., pyrene ${ }^{+}$, the $3.3 \mu \mathrm{m}$ band is missing. The $6.2 \mu \mathrm{m}$ band might be explained by the $\mathrm{C}-\mathrm{C}$ stretching of charged PAHs. However, the covalently bonded system, especially [pyrene $\left.{ }_{2}-\mathrm{Ha}\right]^{+}$agrees the best $(\leqslant 0.1 \mu \mathrm{m})$ with the observations in terms of band positions. The $7.7 \mu \mathrm{m}$ band matches pyrene ${ }^{+}$and [pyrene $\left.{ }_{2}-\mathrm{Ha}\right]^{+}$accurately in comparison to the other species as shown in Figure 7. The other bands cannot be explained by the molecules studied in this work; they might be attributed to larger covalently bonded systems, e.g., a 
recent work shows that the bands 8.6 and $11.2 \mu \mathrm{m}$ might be explained by the dicoronylene cation (Zhen et al. 2018a), which is also a covalently bonded system.

\subsection{Conclusions}

In this work, we performed a theoretical study of molecular dynamics simulations to investigate the reactivity of $\mathrm{PAH}$ radicals and $\mathrm{PAH}$ cations. The simulations show that $\mathrm{PAH}-\mathrm{H}$ is very reactive and can form a covalent bond with a neighbor molecule on a timescale of picoseconds. For a pyrene molecule, all possible H-loss channels are tested-two (pyrene-Ha and pyrene-Hb) out of three isomers lead to the formation of a covalently bonded system, the last one, pyrene-Hc, leads to the formation of a weakly bonded van der Waals dimer. We tested the influence of the charge state for all possible $\mathrm{H}$-loss channels. It turns out that the covalent bond between pyrene$\mathrm{Hx}$ and pyrene can only be formed when one of them is a cation. Furthermore, we studied the $\mathrm{H}_{2}$ losses from pyrene. Three possible $\mathrm{H}_{2}$-loss channels for both neutral and charged systems are tested; however, no covalent bond was observed in the simulation. Instead, only weakly bonded van der Waals dimers are formed.

As mentioned in the introduction, in the ISM, PAH molecules are excited by the absorption of a photon. Several optional pathways might be undertaken to release the excess energy: (1) ionization, (2) IR emission, (3) isomerization, and (4) dissociation, e.g., $\mathrm{H} / \mathrm{H}_{2}$ losses. The molecules necessary for the reactions studied in this work, i.e., $\mathrm{PAH}-\mathrm{H}$ and $\mathrm{PAH}^{+}$, can be efficiently formed following the absorption of single photons with energies below the Lyman limit. The MD simulations show that once these reactive species are produced, a covalently bonded system can be formed rapidly regardless of distance, orientation, and temperature.

For the large PAHs, ionization energies are low, i.e., the larger the $\mathrm{PAH}$, the lower the ionization energies (Holm et al. 2011). Moreover, the dissociation energies for $\mathrm{H}$ loss from neutral PAHs are similar, independent of PAH size (Holm et al. 2011). Therefore, the formation of a covalent bond between $\mathrm{PAH}-\mathrm{H}$ and $\mathrm{PAH}^{+}$ought to be more frequent in comparison to small PAHs.

In addition, this study explains the odd-even pattern of hydrogen loss that is commonly observed in the mass spectra of PAHs for both ion-induced and photoinduced experiments (West et al. 2014; Chen et al. 2015). As the [PAH-H] ${ }^{+}$radicals are so reactive, they catch a neighbor rapidly in the timescale of the experiments $(\sim \mu \mathrm{s})$. Therefore, almost no single $\mathrm{H}$-loss $\mathrm{PAH}$ reaches the detector. However, $[\mathrm{PAH}-2 \mathrm{H}]^{+}$is relatively stable. Hence, a large amount of $[\mathrm{PAH}-2 \mathrm{H}]^{+}$can be detected following the time-of-flight measurements (West et al. 2014; Chen et al. 2015).
This work is supported by the Swedish Research Council (contract No. 2015-06501). The facility is supported by the Swedish National Infrastructure for Computing (project No. SNIC 2018/5-8). The calculations were carried out on Kebnekaise and Abisko located at the High Performance Computing Center North (HPC2N).

\section{ORCID iDs}

Tao Chen (iD https://orcid.org/0000-0003-4145-4300

\section{References}

Allamandola, L., Tielens, A., \& Barker, J. 1985, ApJ, 290, L25 Allamandola, L., Tielens, A., \& Barker, J. 1989, ApJS, 71, 733 Andersson, M. P., \& Uvdal, P. 2005, JPCA, 109, 2937

Andrews, H., Candian, A., \& Tielens, A. 2016, A\&A, 595, A23 Becke, A. D. 1992, JChPh, 96, 2155

Berné, O., \& Tielens, A. G. 2012, PNAS, 109, 401

Chen, T., Gatchell, M., Stockett, M. H., et al. 2015, JChPh, 142, 144305

Delaunay, R., Gatchell, M., Rousseau, P., et al. 2015, JPhCh, 6, 1536

Dyakov, Y. A., Ni, C.-K., Lin, S., Lee, Y., \& Mebel, A. 2006, PCCP, 8, 1404

Ekern, S. P., Marshall, A. G., Szczepanski, J., \& Vala, M. 1998, JPCA, 102, 3498

Frisch, M., Trucks, G., Schlegel, H., et al. 2016, Guassian 16, v.B.01 (Wallingford, CT: Gaussian, Inc.)

Gatchell, M., Delaunay, R., D’Angelo, G., et al. 2017, PCCP, 19, 19665

Grimme, S., Ehrlich, S., \& Goerigk, L. 2011, JCoCh, 32, 1456

Holm, A. I., Johansson, H. A., Cederquist, H., \& Zettergren, H. 2011, JChPh, 134, 044301

Iyengar, S. S., Schlegel, H. B., Millam, J. M., et al. 2001, JChPh, 115, 10291 Jochims, H., Baumgärtel, H., \& Leach, S. 1999, ApJ, 512, 500

Johansson, H. A., Zettergren, H., Holm, A. I., et al. 2011, JChPh, 135, 084304 Lee, C., Yang, W., \& Parr, R. G. 1988, PhRvB, 37, 785

Leger, A., \& Puget, J. 1984, A\&A, 137, L5

Montillaud, J., Joblin, C., \& Toublanc, D. 2013, A\&A, 552, A15

Park, Y. C., \& Lee, J. S. 2006, JPCA, 110, 5091

Rapacioli, M., Calvo, F., Spiegelman, F., Joblin, C., \& Wales, D. 2005, JPCA, 109,2487

Schlegel, H. B., Iyengar, S. S., Li, X., et al. 2002, JChPh, 117, 8694

Schlegel, H. B., Millam, J. M., Iyengar, S. S., et al. 2001, JChPh, 114, 9758

Seitz, F., Zettergren, H., Rousseau, P., et al. 2013, JChPh, 139, 034309

Simon, A., Rapacioli, M., Rouaut, G., Trinquier, G., \& Gadéa, F. 2017, RSPTA, 375, 20160195

Sinnokrot, M. O., Valeev, E. F., \& Sherrill, C. D. 2002, JAChS, 124, 10887

Solano, E. A., \& Mayer, P. M. 2015, JChPh, 143, 104305

Tielens, A. G. G. M. 2008, ARA\&A, 46, 289

Trinquier, G., Simon, A., Rapacioli, M., \& Gadéa, F. X. 2017, MolAs, 7, 37

Tsuzuki, S., Honda, K., Uchimaru, T., Mikami, M., \& Tanabe, K. 2002, JAChS, 124, 104

Vierheilig, A., Chen, T., Waltner, P., et al. 1999, CPL, 312, 349

Wang, Y., Zettergren, H., Rousseau, P., et al. 2014, PhRvA, 89, 062708

West, B., Joblin, C., Blanchet, V., et al. 2012, JPCA, 116, 10999

West, B., Useli-Bacchitta, F., Sabbah, H., et al. 2014, JPCA, 118, 7824

Zettergren, H., Rousseau, P., Wang, Y., et al. 2013, PhRvL, 110, 185501

Zewail, A. H. 2000, JPCA, 104, 5660

Zhen, J., Candian, A., Castellanos, P., et al. 2018a, ApJ, 854, 27

Zhen, J., Castellanos, P., Paardekooper, D. M., et al. 2015, ApJL, 804, L7

Zhen, J., Castellanos, P., Paardekooper, D. M., Linnartz, H., \& Tielens, A. G. 2014, ApJL, 797, L30

Zhen, J., Chen, T., \& Tielens, A. G. 2018b, ApJ, 863, 128 